\title{
Temporal Artery Biopsy in Giant Cell Arteritis
}

\author{
Lindsey B. De Lott, MD, MS, James F. Burke, MD, MS, and Jonathan D. Trobe, MD \\ Department of Neurology, University of Michigan, Ann Arbor (De Lott, Burke, Trobe); Department \\ of Ophthalmology and Visual Sciences, University of Michigan Kellogg Eye Center, Ann Arbor (De \\ Lott, Trobe).
}

\section{In Reply}

We appreciate the thoughtful comments of Grzy-bowski and Stacy regarding the use of laboratory markers in deciding whether to perform TAB in patients with possible GCA. We agree that TAB is important not only for establishing the diagnosis of GCA but also for justifying the use of long-term corticosteroid therapy from which nearly all patients will experience adverse effects. Additionally, TAB may confirm the diagnosis in patients with normal laboratory markers but a suspicious clinical picture.

We are not suggesting that a predictive model using clinical and laboratory information should replace TAB to confirm the diagnosis of GCA, but rather that it may support physician decision making by helping inform physicians which patients may need a biopsy when the decision is not clear cut. The decision to perform a biopsy (and simultaneously start empirical corticosteroid therapy) is often difficult to make, particularly by nonspecialists, and is driven by clinical information and sensitive but nonspecific laboratory information. ${ }^{1}$ This is particularly problematic because studies have shown that physicians are not always adept in interpreting results of multiple diagnostic tests in terms of sensitivity, specificity, and positive predictive value, leading to problems in posttest probability adjustment and errors in medical decision making. ${ }^{2-4}$

Our study suggests that when it comes to integrating demographic and laboratory data into TAB decisions, we have room to improve. Physicians appear to do a good job integrating ESR and CRP information but are not fully using the diagnostic information in age and platelet counts. One potential solution is to develop a clinical risk prediction tool for GCA, not as a replacement for clinical judgment but to help clinicians integrate complex clinical and laboratory information so they can make better medical decisions with their patients.

\section{References}

1. Kermani TA, Schmidt J, Crowson CS, et al. Utility of erythrocyte sedimentation rate and C-reactive protein for the diagnosis of giant cell arteritis. Semin Arthritis Rheum. 2012; 41(6):866-871. [PubMed: 22119103]

Corresponding Author: Lindsey B. De Lott, MD, MS, Department of Ophthalmology and Visual Sciences, University of Michigan Kellogg Eye Center, 1000 Wall St, Ann Arbor, MI 48105 (ldelott@umich.edu).

Conflict of Interest Disclosures: All authors have completed and submitted the ICMJE Form for Disclosure of Potential Conflicts of Interest and none were reported. 
2. Steurer J, Fischer JE, Bachmann LM, Koller M, ter Riet G. Communicating accuracy of tests to general practitioners: a controlled study. BMJ. 2002; 324(7341):824-826. [PubMed: 11934776]

3. Gill CJ, Sabin L, Schmid CH. Why clinicians are natural bayesians. BMJ. 2005; 330(7499):10801083. [PubMed: 15879401]

4. Jackson BR. The dangers of false-positive and false-negative test results: false-positive results as a function of pretest probability. Clin Lab Med. 2008; 28(2):305-319. vii. [PubMed: 18436073] 